Documentation et bibliothèques

DOCUMENTATION BIBLIOTHEQUES

\title{
Documentation et bibliothèques a interrogé ses lecteurs
}

\section{Denis Rousseau}

Volume 22, numéro 2, juin 1976

URI : https://id.erudit.org/iderudit/1055341ar

DOI : https://doi.org/10.7202/1055341ar

Aller au sommaire du numéro

\section{Éditeur(s)}

Association pour l'avancement des sciences et des techniques de la documentation (ASTED)

ISSN

0315-2340 (imprimé)

2291-8949 (numérique)

Découvrir la revue

Citer cet article

Rousseau, D. (1976). Documentation et bibliothèques a interrogé ses lecteurs. Documentation et bibliothèques, 22(2), 55-64. https://doi.org/10.7202/1055341ar
Résumé de l'article

À l'automne 1975, la Rédaction de Documentation et bibliothèques effectuait un sondage auprès des lecteurs de la revue afin, entre autres, de mieux connaître ces derniers, d'évaluer leur degré de satisfaction et d'obtenir des suggestions d'amélioration. Il s'avère que les lecteurs sont généralement satisfaits de la revue dans sa forme et son contenu actuel avec, toutefois, un désir assez clairement exprimé d'articles plus pratiques. Les deux chroniques ( "Bibliothèques et lectures pour jeunes " et " Chronique de la recherche ") ne suscitent pas l'intérêt escompté. Par contre, la section des comptes rendus est particulièrement bien appréciée.
Tous droits réservés (C) Association pour l'avancement des sciences et des techniques de la documentation (ASTED), 1976
Ce document est protégé par la loi sur le droit d'auteur. L'utilisation des services d'Érudit (y compris la reproduction) est assujettie à sa politique d'utilisation que vous pouvez consulter en ligne.

https://apropos.erudit.org/fr/usagers/politique-dutilisation/ 


\title{
Documentation et bibliothèques a interrogé ses lecteurs
}

\author{
Denls Rousseau \\ Secrétaire à la Rédaction
}

A l'automne 1975, la Rédaction de Documentation et bibliothèques effectuait un sondage auprès des lecteurs de la revue afin, entre autres, de mieux connaitre ces derniers, d'évaluer leur degré de satisfaction et d'obtenir des suggestions d'amélioration. II s'avère que les lecteurs sont généralement satisfaits de la revue dans sa forme et son contenu actuel avec, toutefois, un désir assez clairement exprimé d'articles plus pratiques. Les deux chroniques («Bibliothèques et lectures pour jeunes" et "Chronique de la recherche") ne suscitent pas l'intérêt escompté. Par contre, la section des comptes rendus est particulièrement bien appréciée.

In the fall of 1975, the editorial committee of Documentation et bibliothèques carried out a survey amongst its readers in order to get to know them better; to evaluate their degree of satisfaction with the magazine, and to obtain their suggestions for improving it. It appears that the readers are generally satisfied with the present format and content of the magazine, while clearly expressing a desire for articles of a more practical nature. The two chronicles ("Bibliothèques et lectures pour jeunes" et "Chronique de la recherche") do not attract the interest hoped for. On the other hand, the "Comptes rendus" section is particularly well liked.

Durante el otoño de 1975, la Redacción de Documentation et bibliothèques hizo un sondeo de los lectores de la revista para mejor conocerlos, valuar su satisfacción en la revista y obtener sugestiones para aeliorarla. Generalmente, los lectores.se mostraron satisfechos de la revista, con su contenido y su forma pero desearían artículos más prácticos. Las dos crónicas ("Bibliothèques et lectures pour jeunes" $y$ "Chronique de la recherche») no han suscitado el enterés esperado. Pero la sección de cuentas rendidas ha sido muy apreciada.

Une revue doit s'insérer dans une ligne de pensée claire et $s$ 'inspirer de certains principes directeurs tout en tenant compte des problèmes de fonds qui agitent le milieu auquel elle s'adresse. En d'autres termes, elle doit avoir des buts clairement définis et un public aux besoins bien identifiés. Cependant, la réalité étant, par essence, en constante

N.D.R.L. Nous tenons à remercier tout particulièrement Madame Anastassia Khouri-St-Pierre, Messieurs Serge Bertin, Hubert Perron et Réal Messier qui ont soit participé à la compilation des données statistiques, soit aidé à la préparation du texte définitif. évolution, il devient nécessaire de réévaluer périodiquement la pertinence des objectifs et des principes qui guident l'action de la Rédaction.

C'est dans cet esprit qu'a été effectué, à l'automne 1975, un sondage (voir Annexe) auprès des lecteurs de Documentation et bibliothèques, sondage qui avait pour but d'abord de faire le point sur la situation actuelle de la revue, mais surtout de mieux connaître les lecteurs ainsi que leur degré de satisfaction à l'endroit de la revue, et d'obtenir 
des suggestions d'amélioration afin de déterminer, dans une certaine mesure, une nouvelle orientation de la revue.

Le sondage a connu certaines vicissitudes à cause d'une grève qui a affecté le service postal. Un premier envoi n'ayant pas donné un taux satisfaisant de réponses, un rappel a donc dû être fait à la fin de la grève des postes. Le taux final de réponses a été de $53.4 \%$. Le choĩx des répondants, pour l'envoi du questionnaire, s'est effectué selon la méthode de l'échantillonnage: $10 \%$ des noms apparaissant dans la liste d'envoi de l'ASTED ont été retenus.

À noter également qu'à certaines questions (questions 4 et 7 entre autres), quelques répondants ont fourni des deuxièmes et parfois même des troisièmes réponses. Ces réponses ont été ignorées afin de ne pas fausser les pourcentages.

Au moment de l'établissement des pourcentages, nous avons, par contre, tenu compte des "sans-réponse», c'est-à-dire du nombre de répondants qui n'ont pas fourni de réponse à certaines questions. Les pourcentages ont été calculés d'après les réponses obtenues et non d'après le nombre d'envois effectués.

\section{Question 1}

La question 1 portant sur la formation académique était relativement simple; elle avait pour but d'aider à mieux connaître les lecteurs. Voici les résultats.

\begin{tabular}{|l|c|c|c|}
\hline Tableau 1 (Question 1): formation & \multicolumn{3}{|c|}{} \\
\cline { 2 - 4 } & DIPLOMES & ÉTUDIANTS & TOTAL \\
\hline BIBLIOTHÉCONOMIE & $55 \%$ & $5 \%$ & $60 \%$ \\
\hline BIBLIOTECHNIQUE & $27 \%$ & $7 \%$ & $34 \%$ \\
\hline AUTRE & $6 \%$ & - & $6 \%$ \\
\hline TOTAL & $88 \%$ & $12 \%$ & $100 \%$ \\
\hline
\end{tabular}

On notera tout d'abord que $88 \%$ des lecteurs de Documentation et Bibliothèques sont diplômés soit en bibliothéconomie, soit en bibliotechnique ou dans une autre discipline; $12 \%$ sont étudiants en bibliothéconomie ou en bibliotechnique. Cependant, $35 \%$ des lecteurs sont diplômés ou étudiants en bibliotechnique, alors que $60 \%$ le sont en bibliothéconomie, ce qui correspond aux catégories des destinataires du sondage. Ces deux derniers pourcentages ne concordent cependant pas avec les pourcentages de ces mêmes catégories de professionnels membres de l'ASTED. les bibliotechniciens constituant environ $21 \%$ seulement des membres de l'ASTED en 1975. Les résultats du sondage sont donc à in- terpréter en tenant compte de cette légère distorsion. C'est là une conséquence de la méthode utilisée, celle de l'échantillonnage, qu'il nous a été impossible de contrôler sans risquer d'y introduire une trop large part d'arbitraire.

\section{Question 2 et 4}

Les questions 2 et 4 portaient respectivement sur le sexe et le secteur de travail des répondants; elles offraient des affinités de comparaison et le tableau 2 en donne les résultats.

\begin{tabular}{|c|c|c|c|}
\hline \multicolumn{4}{|l|}{ Tableau 2 (Questions 2 et 4): secteur de travail } \\
\hline & FEMMES & HOMMES & TOTAL \\
\hline SERVICES AU PUBLIC & $19 \%$ & $13 \%$ & $32 \%$ \\
\hline SERVICES TECHNIQUES & $13 \%$ & $8 \%$ & $21 \%$ \\
\hline ADMINISTRATION, PLANIFICATION, COORDINATION & $21 \%$ & $12 \%$ & $33 \%$ \\
\hline ENSEIGNEMENT OU RECHERCHE & $6 \%$ & $8 \%$ & $14 \%$ \\
\hline TOTAL & $59 \%$ & $41 \%$ & $100 \%$ \\
\hline
\end{tabular}


Notons tout d'abord qu'à la question 4, sept questionnaires ont dû être rejetés parce que remplis par des étudiants, et que les $4 \%$ des lecteurs qui n'ont pas répondu à cette question (voir Annexe, question 4) ont été ignorés, les pourcentages de ce tableau ayant été calculés à partir des répondants seulement. C'est ce qui explique la différence entre les pourcentages de ce tableau et ceux apparaissant en Annexe, aux questions 2 et 4 . Précisons également que $59 \%$ des répondants sont des femmes et $41 \%$ des hommes, ce qui correspond assez fidèlement au pourcentage des destinataires du questionnaire.

La majorité des répondants œuvrent dans les secteurs "services au public» et «administration, planification, coordination» (32 et $33 \%$ respectivement) alors que seulement $21 \%$ des répondants travaillent dans les services techniques. De plus, $14 \%$ des répondants se consacrent à l'enseignement et à la recherche, ce qui est assez conforme à la réalité, l'enseignement et la recherche étant nécessairement le lot d'une minorité de spécialistes de la documentation.

\section{Question 3}

La troisième question qui portait sur le groupe d'âge a démontré que les lecteurs de
Documentation et bibliothèques se répartissent assez uniformément entre toutes les catégories d'âge avec, toutefois, une légère prédominance des 25 à 34 ans (33\%); les moins de 25 ans constituent $23 \%$ des répondants, ceux de 35 à 44 ans, $22 \%$ et ceux de 45 ans et plus, $22 \%$ également.

Ces quatre premières questions avaient pour but de connaître les lecteurs de Documentation et bibliothèques. Si nous avions à dresser un portrait du lecteur-type de la revue, nous pourrions affirmer que c'est un bibliothécaire féminin d'environ une trentaine d'années œuvrant dans les services au public ou occupant un poste de cadre moyen.

\section{Question 5}

Les questions 5 et 6 visaient à faire connaître le comportement des répondants en regard de la lecture des revues professionnelles. Notons en passant que tous les répondants ont fourni une réponse à la question 5 , alors que le taux des sans-réponse s'élève à $13 \%$ à la question 6.

Examinons tout d'abord le tableau 3 qui illustre la question 5 portant spécifiquement sur la fréquence et l'habitude de lecture de Documentation et bibliothèques.

Tableau 3 (Questions 5.1 et 5.2): fréquence et habitude de lecture de Documentation et bibliothèques

\begin{tabular}{|c|c|c|c|c|c|}
\hline \multicolumn{6}{|c|}{ ARTICLES } \\
\hline FREQUENCE HABITUDE & TOUS & MAJORITE & QUELQUES & AUCUN & TOTAL \\
\hline TOUJOURS & $11 \%$ & $25 \%$ & $20 \%$ & - & $56 \%$ \\
\hline SOUVENT & - & $7 \%$ & $11 \%$ & - & $18 \%$ \\
\hline OCCASIONNELLEMENT & - & $4 \%$ & $16 \%$ & - & $20 \%$ \\
\hline RAREMENT & - & - & $6 \%$ & - & $6 \%$ \\
\hline JAMAIS & - & - & - & - & - \\
\hline TOTAL & $11 \%$ & $36 \%$ & $53 \%$ & - & $100 \%$ \\
\hline
\end{tabular}

On remarque immédiatement que tous les répondants lisent au moins, à l'occasion, quelques articles de la revue, la majorité se situant nettement dans les catégories "toujours" et "Souvent" qui totalisent, à elles seules, $74 \%$ des répondants.
Peu de lecteurs lisent tous les articles (11\%) ce qui est normal, chacun s'attardant généralement aux articles qui touchent plus particulièrement son domaine d'activité et ses intérêts. $36 \%$ des répondants lisent habituellement la majorité des articles et $11 \%$ 
lisent tous les articles, ce qui équivaut à un bloc de lecteurs assidus de $47 \%$ des répondants. Toutefois, même si la majorité des répondants ne lisent que quelques articles (53\%), il est également vrai d'affirmer que la majorité, soit $56 \%$, lisent toujours la revue, mais selon des modalités diverses allant de tous les articles à quelques-uns.

On remarque également une nette tendance à l'effet que les gens lisant plus régulièrement la revue (catégorie "toujours») sont aussi ceux qui lisent le plus grand nombre d'articles de chaque numéro ( 11 et $25 \%$ ). Bref, plus on lit souvent la revue, plus on la lit intégralement.

Aucun des répondants n'a indiqué ne jamais lire la revue, ou ne lire aucun article de la revue, ce qui revient au même. Documentation et bibliothèques serait donc une revue lue, à une fréquence variée mais soutenue, ce qui prouve l'intérêt qu'elle suscite et son utilité sur la scène de la bibliothéconomie québécoise. Toutefois, les non-répondants pourraient assombrir quelque peu cette perspective puisqu'ils pourraient être les moins intéressés à la revue ou ceux qui la lisent le moins. C'est là une variable qu'il nous est malheureusement impossible de contrôler, ces derniers n'ayant pas répondu au questionnaire.

\section{Question 6}

Cette question qui portait sur l'habitude de lecture d'autres revues d'intérêt professionnel révèle que le Bulletin de l'Unesco à l'intention des bibliothèques est la plus lue parmi les autres revues puisqu'il suscite l'intérêt de $42 \%$ des répondants. En deuxième lieu vient Argus (38\%) et en troisième position Canadian Library Journal (25\%). II semble donc acquis que la majorité des lecteurs de Documentation et bibliothèques lisent également au moins une autre revue d'intérêt professionnel (entendons "professionnel» au sens large).

A la sous-question "Lisez-vous d'autres revues de langue française?" $35 \%$ des sujets ont répondu affirmativement et les revues le plus fréquemment mentionnées sont, par ordre décroissant, le Bulletin des bibliothèques de France, le Bulletin du livre et Vient de paraître, le Bulletin de bibliographie et plusieurs autres mentionnées une seule fois (environ une dizaine). A noter que le taux de non-répondants, pour cette suus-question, s'élève à $25 \%$, ce qui laisse croire que le quart des répondants ne lisent possiblement pas d'autres revues de langue française que les deux mentionnées à la première partie de la question 6 .
En ce qui concerne les autres revues de langue anglaise, le pourcentage des non-lecteurs est, cette fois, plus élevé que celui des lecteurs, puisque la proportion est de $36 \%$ contre $33 \%$, les sans-réponse comptant pour $18 \%$. Les revues le plus fréquemment mentionnées sont, toujours selon un ordre décroissant, Special Libraries, Library Trends, College and Research Libraries et Library Journal (ces trois derniers titres étant sur un pied d'égalité), Wilson Library Bulletin, Ontario Library Review et Bulletin of the Medical Library Association qui, tous trois, se partagent la même position, et une quinzaine d'autres revues mentionnées une seule fois.

Si nous additionnons les sans-réponse (à la question générale (13\%) et aux sous-questions (25 et 18\%) et ceux qui ont répondu négativement aux deux sous-questions, on obtient des chiffres qui permettent de croire que la majorité des lecteurs de Documentation et bibliothèques, s'ils lisent une ou des revues parmi les trois revues mentionnées, n'en lisent définitivement pas d'autres (de langue française: $65 \%$ et de langue anglaise: $67 \%$ ), les "Sans-réponse" tant à la question générale qu'aux sous-questions étant considérés comme ne lisant pas d'autres revues. Ce dernier résultat accentue encore davantage l'importance, pour Documentation et bibliothèques, d'avoir un contenu et un niveau variés afin de répondre aux besoins et aux intérêts du plus grand nombre possible de lecteurs.

II s'ensuit que Documentation et bibliothèques peut être considérée par beaucoup de ses lecteurs comme un instrument d'éducation permanente ou de recyclage, d'où influence directe sur le contenu de la revue (sujets traités, nature et niveau des articles).

\section{Question 7}

Les réponses à la question 7 sont suffisamment significatives pour conclure à la popularité de certains types d'articles et à la non-popularité de certains autres. Ainsi les articles jugés les plus intéressants sont les suivants:

1. "Les bibliotechniciens dans un culde-sac; à qui la faute?» (76\%)

2. «Bibliotechnique: techniques de la documentation?" (64\%)

3. "Les bibliothèques universitaires canadiennes, dix ans après» (49\%)

4. "La bande dessinée a-t-elle droit de cité à la bibliothèque?" (46\%)

5. "La documentation et ses langages" (45\%) 
6. "La documentation, d'hier à demain" (44\%)

7. "L'automatisation de la bibliographie nationale du Canada: Canadiana" (1re partie) (44\%)

8. "Les besoins de personnel professionnel dans les bibliothèques publiques du Québec" (40\%)

On notera tout d'abord que les articles portant sur la bibliotechnique sont de loin ceux qui ont le plus intéressé les lecteurs $(76 \%$ et $64 \%$ ) et, par conséquent, ceux pour lesquels le pourcentage de "non-lu» est le plus faible $(6 \%$ et $16 \%)$. Ces résultats sont à interpréter en tenant compte des commentaires émis à la question 1 , à savoir que le nombre de bibliotechniciens répondants est un peu plus élevé que le pourcentage réel de bibliotechniciens membres de l'ASTED.

Parmi ces huit articles jugés les plus intéressants, six sont à tendance nettement pratique alors que deux sont plutôt théoriques ("La documentation et ses langages" et "La documentapion, d'hier à demain"). On serait tenté de conclure que la pratique est préférée par la majorité des lecteurs, quoique ce ne soit pas là un absolu: la théorie a aussi sa place. Cette constatation est à rapprocher des résultats obtenus à la question 10 où $66 \%$ des répondants considèrent les articles de Documentation et bibliothèques suffisamment théoriques alors que seulement $42 \%$ les jugent suffisamment pratiques et que $30 \%$ affirment, pour leur part, qu'ils ne soni pas assez pratiques.

La colonne des "utile» est moins significative, les pourcentages étant trop faibles pour permettre de tirer des conclusions très précises. Peut-être y a-t-il eu une certaine confusion entre les termes "intéressant" et "utile", plusieurs ayant inclu "utile» dans le précédent. Mentionnons néanmoins les pourcentages les plus significatifs:

1. "Littérature de jeunesse au Canada français - édition 1973" (27\%)

2. "Catalographie: le comité des noms géographiques" (26\%)

3. "Un service de bibliothèque aux handicapés physiques et visuels» $(25 \%)$

Il est intéressant de remarquer que ces trois titres coiffent des articles plutôt pratiques. Peu d'articles donc ont été jugés utiles au sens de l'application pratique, ce qui est à rapprocher également des $30 \%$ de la question 10 qui soutiennent que les articles ne sont pas assez pratiques.

Les articles ayant présenté le moins d'intérêt sont:

1. "La femme, espèce humaine?" (15\%)

2. "L'agrément américain et nous" $(7 \%)$

3. «A la bibliothèque de l'Université Laval, la Référence fait signe aux usagers" $(7 \%)$

4. "Le "Cas Blyton" (7\%)

5. "L'automatisation de la bibliographie nationale du Canada: Canadiana (2e partie)" $(7 \%)^{1}$

6. "Mémoire à la Commission parlementaire de la Justice de l'Assemblée nationale du Québec" (7\%)

Tous ces pourcentages s'avèrent néanmoins très bas, sauf le premier. A l'analyse de ces titres, pourrait-on affirmer que les articles suscitant une certaine polémique (ce qui est le cas de quatre de ces six articles) rebutent de prime abord et, par voie de corollaire, que les lecteurs de Documentation et bibliothèques ne seraient pas très friands de sujets controversés...?

Les pourcentages obtenus à la question "Non-lu» sont beaucoup plus probants:

1. "Le "Cas Blyton" (54\%)

2. "L'agrément américain et nous" (49\%)

3. "Analyse opérationnelle dans les bibliothèques de l'Université de Sherbrooke" (49\%)

4. "Mémoire à la Commission parlementaire de la Justice de l'Assemblée nationale du Québec» (49\%)

5. "Les publications officielles québécoises. Le journal des Débats" (45\%)

6. «PRECIS I: Preserved Context Indexing System» (45\%)

7. «Bibliothèques et archives du TiersMonde: problèmes et perspectives" (45\%)

Si nous additionnons les pourcentages de "Aucun intérêt" et "Non-lu», en prenant pour acquis que ceux qui n'ont pas lu ces textes

1. Ce pourcentage nous laisse un peu perplexe puisque la première partie de cet article a suscité un intérêt plus marqué. II est cependant toujours possible que les différentes parties d'un texte retiennent l'attention à des degrés divers, tout dépendant de la facette ou des éléments du problème qui sont traités. 
n'y trouvaient probablement aucun intérêt, on obtient des totaux tous égaux ou supérieurs à $49 \%$, ce qui revient à dire que la moitié des répondants ou plus n'ont pas apprécié les titres ci-haut mentionnés. On y retrouve plusieurs articles théoriques, tels ceux traitant de l'agrément américain, de la liberté intellectuelle et de l'analyse opérationnelle. II est un peu allarmant de constater que si peu de lecteurs s'intéressent à des questions aussi fondamentales que, par exemple, la liberté intellectuelle et l'analyse opérationnelle. Serait-ce que le milieu de la bibliothéconomie québécoise n'est pas prêt à aborder franchement et collectivement des problèmes ou des questions qui débordent les cadres du quotidien? Le débat reste ouvert.

II ressort de l'analyse des résultats de cette question une autre constatation d'importance concernant les chroniques. En examinant attentivement l'intérêt qu'elles ont suscité, on doit se rendre à l'évidence que peu d'entre elles ont vraiment retenu l'attention des lecteurs à l'exception de celle traitant de la bande dessinée dont voici les résultats: "Intéressant»: $46 \%$; "Utile»: $16 \%$, ce qui donne $62 \%$ de lecteurs intéressés par cette chronique. Ces résultats généraux permettent de remettre en question, peut-être pas l'existence même des chroniques, mais à tout le moins leur fréquence: quatre chroniques en littérature de jeunesse et un nombre égal de chroniques portant sur la recherche, chaque année, c'est peut-être trop considérable présentement.

\section{Question 8}

Cette question portait sur les comptes rendus: leur fréquence de lecture (8.1), leur nombre (8.2) et leur qualité (8.3). Des résultats de la première question, on peut déduire que la très grande majorité des sujets lisent au moins quelques-uns des comptes rendus puisque seulement $9 \%$ des répondants affirment ne lire aucun compte rendu.

Les résultats de la deuxième question sont également concluants car $69 \%$ des lecteurs considèrent que les comptes rendus sont en nombre suffisant.

Quant à la troisième question, elle permet de constater que $56 \%$ des répondants qualifient d'excellente et de très bonne la qualité des comptes rendus, alors que $4 \%$ seulement la jugent médiocre. De fait, il y a très peu de répondants qui évaluent la qualité des comptes rendus comme excellente $(9 \%)$, la très grande majorité $(82 \%)$ la situant entre bonne et très bonne.

Tableau 4 (Questions 8.1 et 8.2): évaluation des comptes rendus

(habitude de lecture et nombre)

\begin{tabular}{|c|c|c|c|c|}
\hline HABITUDE & ESTIMATION & SUFFISANT & INSUFFISANT & TOTAL \\
\hline MAJORITE & & $18 \%$ & $16 \%$ & $34 \%$ \\
\hline QUELQUES-UNS & & $50 \%$ & $10 \%$ & $60 \%$ \\
\hline AUCUN & & $4 \%$ & $2 \%$ & $6 \%$ \\
\hline TOTAL & & $72 \%$ & $28 \%$ & $100 \%$ \\
\hline
\end{tabular}

Pour la préparation du tableau 4, quatre questionnaires ont dû être éliminés du fait qu'ils ne comportaient aucune réponse à la question 8.2 portant sur le nombre des comptes rendus. C'est ce qui explique encore une fois la différence entre les pourcentages obtenus dans ce tableau et ceux apparaissant en Annexe aux questions 8.1 et 8.2.

Ce tableau permet de conclure que près de la moitié des répondants lisant la majorité des comptes rendus (34\%) considèrent leur nombre insuffisant dans une proportion de $16 \%$; par contre, la grande majorité des répondants ne lisant que quelques-uns ou aucun des comptes rendus (66\%) estiment leur nombre suffisant (54\%). En somme, plus les gens lisent les comptes rendus, plus ils souhaitent l'augmentation de leur nombre.

En résumé, les comptes rendus s'avèrent une section lue assidûment et leur qualité oscille entre bonne et très bonne, voire même excellente; leur nombre cependant aurait 
peut-être avantage à être augmenté. Cette partie de Documentapion et bibliothèques est donc particulièrement bien appréciée des lecteurs.

\section{Question 9}

Cette question qui portait sur la présentation matérielle de la revue a permis de constater que le degré de satisfaction des lecteurs va décroissant en passant du format à la typographie puis au graphisme: le format est jugé excellent par $60 \%$ des lecteurs, la typographie excellente et très bonne par $38 \%$ des lecteurs dans les deux cas et le graphisme, quant à lui, est jugé très bon et bon par 35 et $29 \%$ des lecteurs. Aucun des répondants ne considère médiocre le format ou la typographie. Par contre, $5 \%$ des répondants jugent que le graphisme est médiocre. II y a donc là l'indication d'une amélioration possible à apporter à la présentation visuelle. Néanmoins, la majorité des appréciations, et cela vaut pour les trois sujets analysés, se situe aux niveaux "excellent" et "très bon".

\section{Question 10}

Cette question semble avoir été mal perçue par plusieurs des répondants, le pourcentage des sans-réponse oscillant entre 4 et $15 \%$. La caractéristique "académique" a tout particulièrement fait hésiter bon nombre de lecteurs. En outre, au cours de la compilation du questionnaire, nous avons relevé certaines incongruités, tels ces répondants qui affirmaient d'un même souffle que la revue était à la fois assez générale et assez spécialisée.

Compte tenu de ces réserves, la compilation des réponses permet néanmoins de conclure que la majorité des lecteurs ont répondu "suffisamment" à quatre des cinq aspects de cette question, soit "théorique", "générale", "spécialisée" et "académique». Pour ce qui est du cinquième aspect, la dimension pratique, seulement $42 \%$ des répondants estiment les articles "suffisamment" pratiques alors que $30 \%$ croient qu'ils ne le sont pas assez. Cette insatisfaction assez nettement exprimée, qui doit aussi être analysée en regard des commentaires émis à la question 1 , rejoint l'appréciation des articles faite à la question 7 . Doit-on en conclure que Documentation et bibliothèques devrait traiter davantage de thèmes éminemment pratiques au détriment de sujets à contenu plus théorique...?

Cette demande d'articles plus pratiques nous amène à nous poser la question suivan- te: une revue telle que Documentation et bibliothèques qui vise à atteindre un certain niveau scientifique doit-elle surtout coller aux besoins immédiats des lecteurs en leur présentant des cas concrets, des expériences vécues, ou plutôt tenter de s'élever légèrement au-dessus de l'immédiat (sans toutefois délaisser complètement cette actualité des problèmes quotidiens) afin de faire avancer la discipline? La science étant cumulative et non répétitive, il importe peut-être d'explorer des avenues nouvelles et ce, tant au niveau de la théorie que de la pratique.

\section{Conclusion}

Les objectifs de ce sondage ont été atteints et les résultats, on l'aura deviné, posent suffisamment de questions pour donner matière à réflexion. II semble assez évident que l'accent ne devra pas être mis sur les articles très théoriques et ce, sans toutefois nuire à l'orientation scientifique déjà imprimée à la revue. C'est là un équilibre difficile à maintenir et qui devra préoccuper constamment la Rédaction.

Le nombre de comptes rendus pourra être augmenté, sans que cette démarche constitue cependant une priorité.

Il y aurait peut-être lieu, à l'avenir, de développer une formule de compte rendu qui s'apparente au "review article», c'est-à-dire un compte rendu assez élaboré qui, à partir d'un ouvrage donné, fasse le point sur une question ou un problème particulier.

Quant aux chroniques, étant donné l'intérêt mitigé qu'elles suscitent, la Rédaction a décidé de les maintenir, mais selon le principe de l'alternance, en ce sens que chaque livraison de Documentation et bibliothèques ne contiendra qu'une seule chronique, soit "Bibliothèques et lectures pour jeunes". soit "Chronique de la recherche"; chacune des chroniques apparaîtra donc au sommaire deux fois par année au lieu de quatre comme c'est le cas présentement.

Ces améliorations, et quelques autres, ne doivent pas faire oublier une des conclusions majeures de ce sondage: Documentation et bibliothèques est une revue très appréciée des lecteurs dont la majorité des articles intéressent grandement les répondants et qui constitue, peut-être à son insu, un instrument de recyclage et d'éducation permanente. 
ANNEXE

\section{Documentation et bibliothèques}

\section{Sondage}

\section{Septembre 1975}

Le présent sondage est réalisé par le comité de rédaction de Documentation et bibliothèques en vue de mieux connaître les lecteurs, d'évaluer leur degré de satisfaction et de déterminer l'orientation de la revue. Veuillez avoir l'amabilité de le retourner dûment rempli (en cochant les cases appropriées) à:

\section{Documentation et bibliothèques}

360 , rue Le Moyne

Montréal, Qué.

1. Quelle est votre formation académique?

- diplômé en bibliothéconomie

- diplômé en bibliotechnique

- diplômé dans une autre discipline spécifier

- étudiant en bibliothéconomie

- étudiant en bibliotechnique

- étudiant dans une autre discipline spécifier

2. Quel est votre sexe?

$$
\begin{aligned}
& \text { - masculin } \\
& \text { - féminin }
\end{aligned}
$$

3. A quel groupe d'âge appartenez-vous?

$$
\begin{aligned}
& \text { - moins de } 25 \text { ans } \\
& -25 \text { à } 34 \text { ans } \\
& -35 \text { à } 44 \text { ans } \\
& -45 \text { et plus }
\end{aligned}
$$

4. Quel est votre secteur de travail (les étudiants voudront bien s'abstenir de répondre à cette question)? (S.R.:* $4 \%$ )

$$
\begin{aligned}
& \text { - services au public } \\
& \text { - services techniques } \\
& \text { - administration, planification, coordination } \\
& \text { - enseignement ou recherche }
\end{aligned}
$$

\section{Lisez-vous Documentation et bibliothèques?}

5.1 Fréquence de lecture:

$$
\begin{aligned}
& \text { - toujours } \\
& \text { - souvent } \\
& \text { - occasionnellement } \\
& \text { - rarement } \\
& \text { - jamais }
\end{aligned}
$$

5.2 Habitude de lecture:

$$
\begin{aligned}
& \text { - tous les articles } \\
& \text { - la majorité des articles } \\
& \text { - quelques articles } \\
& \text { - aucun article }
\end{aligned}
$$


6. Lisez-vous d'autres revues d'intérêt professionnel? (S.R.: 13\%)

- Canadian Library Journal 25\%

- Argus $38 \%$

- Bulletin de I'Unesco à l'intention des bibliothèques $\quad 42 \%$

- revues de langue française: oui \} (S.R.: 25\%) $35 \%$

si oui, spécifier les plus importantes

- revues de langue anglaise: $\left.\begin{array}{l}\text { oui } \\ \text { non }\end{array}\right\}$ (S.R.: 18\%)

7. Évaluation des articles:

Vol. 20, no 3 (septembre 1974)

- L'agrément américain et nous

- La documentation et ses langages

- La documentation, d'hier à demain

- Services documentaires et sciences de l'information

- Littérature de jeunesse au Canada français - édition 1973

- A la bibliothèque de l'Université Laval, la Référence fait signe aux usagers

Vol. 20, no 4 (décembre 1974)

- Les publications officielles québécoises. Le journal des Débats

- Les besoins de personnel professionnel dans les bibliothèques publiques du Québec

- L'automatisation de la bibliographie nationale du Canada: Canadiana (1 re partie)

- Pour une analyse "marketing" de la bibliothèque

- Le "Cas Blyton"

- La recherche en matière de classification: état de la question

Vol. 21, no 1 (mars 1975)

- Mot de la Rédaction

- La femme, espèce humaine?

- PRÉCIS I: Preserved Context Indexing System

- L'automatisation de la bibliographie nationale du Canada: Canadiana (2e partie)

- Les bibliothèques universitaires canadiennes, dix ans après...

- Analyse opérationnelle dans les bibliothèques de l'université de Sherbrooke

- La nouvelle bibliothèque pour jeunes de Saint-Léonard

- Catalographie: le comité des noms géographiques

Vol. 21, no 2 (Juin 1975)

- Les bibliotechniciens dans un cul-desac; à qui la faute?

- Mémoire à la Commission parlementaire de la Justice de l'Assemblée nationale du Québec

\begin{tabular}{|c|c|c|c|c|}
\hline $\begin{array}{c}\text { Inté- } \\
\text { ressant }\end{array}$ & Utile & $\begin{array}{l}\text { Aucun } \\
\text { intérêt }\end{array}$ & $\begin{array}{l}\text { Non } \\
\text { lu }\end{array}$ & S.R. \\
\hline $15 \%$ & $18 \%$ & $7 \%$ & $49 \%$ & $11 \%$ \\
\hline $45 \%$ & $24 \%$ & & $20 \%$ & $11 \%$ \\
\hline $44 \%$ & $13 \%$ & $3 \%$ & $31 \%$ & $9 \%$ \\
\hline $39 \%$ & $24 \%$ & $2 \%$ & $26 \%$ & $9 \%$ \\
\hline $22 \%$ & $27 \%$ & & $42 \%$ & $9 \%$ \\
\hline $29 \%$ & $22 \%$ & $7 \%$ & $29 \%$ & $13 \%$ \\
\hline $16 \%$ & $22 \%$ & $4 \%$ & $45 \%$ & $13 \%$ \\
\hline $40 \%$ & $24 \%$ & $5 \%$ & $24 \%$ & $7 \%$ \\
\hline $44 \%$ & $13 \%$ & $5 \%$ & $29 \%$ & $9 \%$ \\
\hline $29 \%$ & $20 \%$ & $2 \%$ & $36 \%$ & $13 \%$ \\
\hline $22 \%$ & $2 \%$ & $7 \%$ & $54 \%$ & $15 \%$ \\
\hline $29 \%$ & $22 \%$ & & $36 \%$ & $13 \%$ \\
\hline $22 \%$ & $21 \%$ & $5 \%$ & $38 \%$ & $14 \%$ \\
\hline $33 \%$ & $11 \%$ & $15 \%$ & $28 \%$ & $13 \%$ \\
\hline $22 \%$ & $16 \%$ & $4 \%$ & $45 \%$ & $13 \%$ \\
\hline $38 \%$ & $14 \%$ & $7 \%$ & $31 \%$ & $10 \%$ \\
\hline $49 \%$ & $9 \%$ & $4 \%$ & $27 \%$ & $11 \%$ \\
\hline $20 \%$ & $16 \%$ & $4 \%$ & $49 \%$ & $11 \%$ \\
\hline $33 \%$ & $22 \%$ & $2 \%$ & $40 \%$ & $3 \%$ \\
\hline $16 \%$ & $26 \%$ & $5 \%$ & $40 \%$ & $13 \%$ \\
\hline $76 \%$ & $11 \%$ & $2 \%$ & $6 \%$ & $5 \%$ \\
\hline $11 \%$ & $18 \%$ & $7 \%$ & $49 \%$ & $15 \%$ \\
\hline
\end{tabular}


- Bibliotechnique: techniques de la documentation?

- Bibliothèques et archives du TiersMonde: problèmes et perspectives

- La banque dessinée a-t-elle droit de cité à la bibliothèque?

- Un service de bibliothèque aux handicapés physiques et visuels

$\begin{array}{ll}64 \% & 13 \% \\ 27 \% & 13 \% \\ 36 \% & 25 \% \\ & \\ & \\ & \\ & \\ & \end{array}$

$13 \%$

$16 \%$

$45 \%$

$29 \%$

$29 \%$

$25 \%$
$7 \%$

$11 \%$

$5 \%$

$9 \%$

8. Évaluation des comptes rendus:

8.1. Habitude de lecture: lisez-vous:

$$
\begin{aligned}
& \text { - la majorité des comptes rendus } \\
& \text { - quelques-uns des comptes rendus } \\
& \text { - aucun des comptes rendus }
\end{aligned}
$$

8.2 Compte tenu de l'existence de la chronique "Livres reçus" des Nouvelles de l'ASTED, estimezvous que les comptes rendus sont en nombre

$$
\text { - } \left.\begin{array}{l}
\text { suffisant } \\
\text { - insuffisant }
\end{array}\right\} \text { (S.R.: 7\%) }
$$

8.3 La qualité des comptes rendus vous semble-t-elle

$$
\left.\left.\begin{array}{l}
\text { - excellente } \\
- \text { très bonne } \\
- \text { bonne } \\
- \text { médiocre }
\end{array}\right\} \text { (S.R.: } 5 \%\right)
$$

9. Précisez votre évaluation globale de la présentation matérielle de Documentation et bibliothèques:

$$
\begin{aligned}
& \text { - format } \\
& \text { - typographie } \\
& \text { - graphisme }
\end{aligned}
$$

$\begin{array}{cc}\text { Excellent } & \text { Très bon } \\ 60 \% & 27 \% \\ 38 \% & 38 \% \\ 27 \% & 35 \%\end{array}$

Bon
$11 \%$
$22 \%$
$29 \%$

Médiocre

S.R.

$22 \%$

$\begin{array}{rl} & 2 \% \\ & 2 \% \\ 5 \% & 4 \%\end{array}$

10. Comment qualifiez-vous la teneur des articles de Documentation et bibliothèques?

\section{Suffisam-}

$$
\begin{aligned}
& \text { - théorique } \\
& \text { - pratique } \\
& \text { - générale } \\
& \text { - spécialisée } \\
& \text { - académique }
\end{aligned}
$$

\section{Très}

$20 \%$

$24 \%$

$11 \%$

$17 \%$

$19 \%$

\section{ment}

$66 \%$

$42 \%$

$68 \%$

$62 \%$

$58 \%$
Pas assez

$4 \%$

$30 \%$

$8 \%$

$13 \%$

$8 \%$
S.R.

$10 \%$

$4 \%$

$13 \%$

$8 \%$

$15 \%$

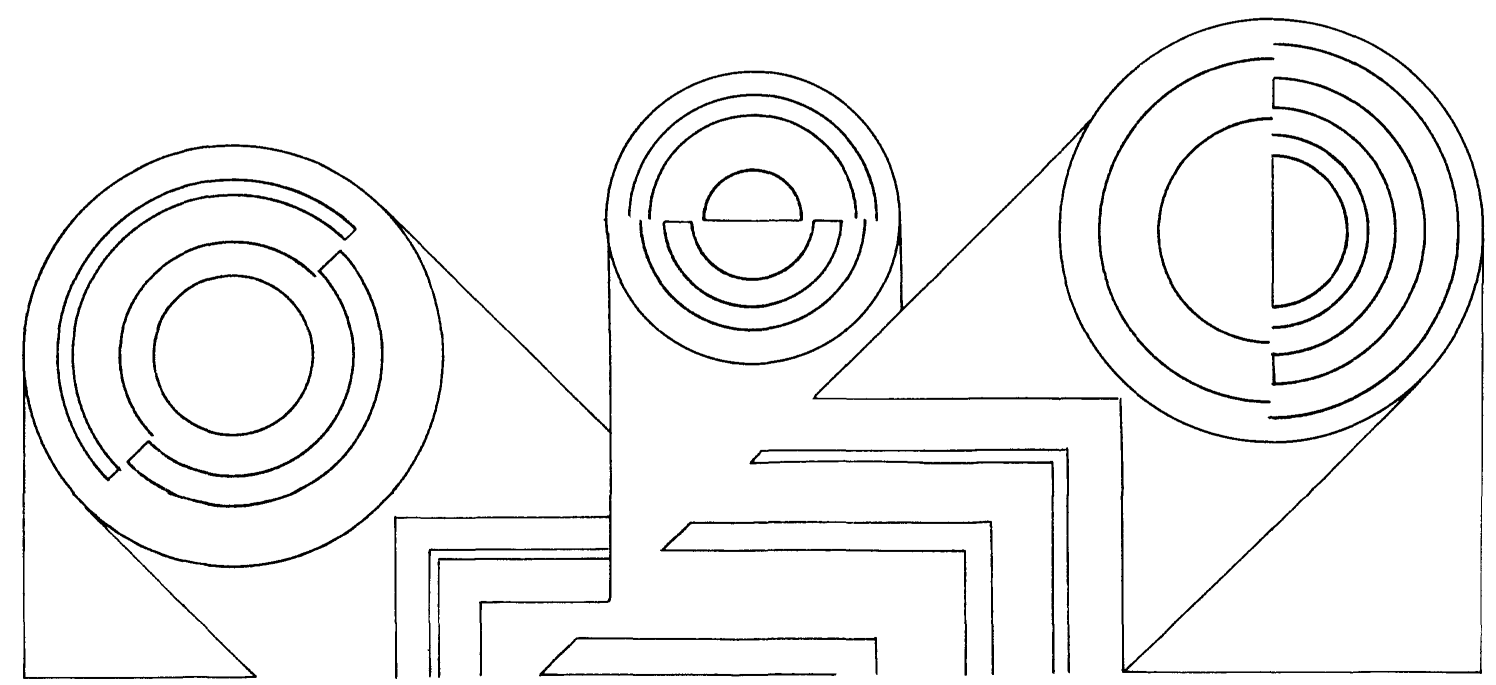

